\title{
GENERALIZED CLUSTER SETS OF REAL FUNCTIONS
}

\author{
JACEK JĘDRZEJEWSKI — StAnisŁaW KowAlCZYK
}

\begin{abstract}
Limit numbers of real functions were introduced into considerations in many articles. In the present paper, new ideas of generalized limit numbers and some first properties of such topics can be found. In particular, connections with generalized topologies are studied.
\end{abstract}

\section{Introduction}

We will consider only real functions defined in an open interval, let it be $I$. When we use topological terminology, then it is applied in the sense of natural topology in the set of real numbers (or in its subsets).

Limit numbers of a real function defined in subsets of $\mathbb{R}$ have been considered in many articles by many mathematicians. Starting from the classical result of $\mathrm{W}$. H. Y o u n $[12$ concerning asymmetry of functions through problems of usual limit numbers [5], 8], approximate limit numbers discussed by M. K u l backa [7, L. B elowska [1, W. Wilczyński [13] and others, problems of qualitative limit numbers (W. W i l c z y ńs k i [14]), $\mathscr{B}$-limit numbers [3], [4, [6], up to a big monograph on local systems by B. S. T h o m s o n [11.

The so-called generalized topological spaces have been introduced into many mathematical articles, following the idea which was introduced by Á. C s ás z ár in [2]. In view of this idea, it is possible to consider generalized continuity and related problems. We want to consider some problems dealing with such a field.

\section{Basic notions and properties}

Let us start from reminding some of definitions from the article [3].

If $E$ is a subset of the reals $(\mathbb{R})$ and $x \in \mathbb{R}$, then

$$
-E=\{x:-x \in E\} \quad \text { and } \quad E+x=x+E=\left\{y \in \mathbb{R}: \exists_{a \in E}(y=a+x)\right\} .
$$

(c) 2015 Mathematical Institute, Slovak Academy of Sciences. 2010 Mathematics Subject Classification: 54C30.

Keyw ords: generalized limit numbers, generalized topology. 
Definition ([3]). Let $\mathfrak{B}_{0}^{+}$denote a class of nonempty subsets of $\mathbb{R}$ which fulfils the following conditions:

$$
\begin{aligned}
E \in \mathfrak{B}_{0}^{+} \wedge t>0 & \Longrightarrow E \cap(0, t) \in \mathfrak{B}_{0}^{+}, \\
E_{1} \cup E_{2} \in \mathfrak{B}_{0}^{+} & \Longleftrightarrow\left(E_{1} \in \mathfrak{B}_{0}^{+} \vee E_{2} \in \mathfrak{B}_{0}^{+}\right) .
\end{aligned}
$$

Next, let

$$
\mathfrak{B}_{0}^{-}=\left\{E \subset \mathbb{R}:-E \in \mathfrak{B}_{0}^{+}\right\} .
$$

For every $x \in \mathbb{R}$, let

$$
\mathfrak{B}_{x}^{+}=\left\{E \subset \mathbb{R}: E-x \in \mathfrak{B}_{0}^{+}\right\} \quad \text { and } \quad \mathfrak{B}_{x}^{-}=\left\{E \subset \mathbb{R}: E-x \in \mathfrak{B}_{0}^{-}\right\},
$$

Finally, let us assume that

$$
\mathfrak{B}_{x}=\mathfrak{B}_{x}^{+} \cup \mathfrak{B}_{x}^{-} \quad \text { and } \quad \mathfrak{B}=\left\{\mathfrak{B}_{x}: x \in \mathbb{R}\right\} .
$$

Definition ([3]). A number $g$ is called $\mathfrak{B}$-limit number of a function $f: I \longrightarrow \mathbb{R}$ at a point $x_{0}$ from the right side if

$$
\{x \in I:|f(x)-g|<\varepsilon\} \in \mathfrak{B}_{x_{0}}^{+}
$$

for each positive real number $\varepsilon$.

Infinity is called $\mathfrak{B}$-limit number of a function $f: I \longrightarrow \mathbb{R}$ at a point $x_{0}$ from the right side if

$$
\{x \in I: f(x)>\varepsilon\} \in \mathfrak{B}_{x_{0}}^{+}
$$

for each positive real number $\varepsilon$.

Minus infinity is called $\mathfrak{B}$-limit number of a function $f: I \longrightarrow \mathbb{R}$ at a point $x_{0}$ from the right side if

$$
\{x \in I: f(x)<-\varepsilon\} \in \mathfrak{B}_{x_{0}}^{+}
$$

for each positive real number $\varepsilon$.

The above mentioned definitions generalize classic limit numbers of real functions. Some properties of those $\mathfrak{B}$-limit numbers were studied in [3] and generalized for some topological case in [4.

In the present article, we are going to give more general approach to that problem.

Definition 1. For each real $x$, let $\mathscr{B}_{x}^{+}$denote a nonempty class of nonempty subsets of $\mathbb{R}$ which fulfils the following conditions:

$$
\begin{aligned}
& \text { if } E \in \mathscr{B}_{x}^{+} \text {and } t>0, \text { then } E \cap(x, x+t) \in \mathscr{B}_{x}^{+} \text {, } \\
& \text { if } E \in \mathscr{B}_{x}^{+} \text {and } F \supset E \text {, then } F \in \mathscr{B}_{x}^{+} .
\end{aligned}
$$

For each real $x$, let $\mathscr{B}_{x}^{-}$denote a nonempty class of nonempty subsets of $\mathbb{R}$ which fulfils the following conditions:

$$
\begin{aligned}
& \text { if } E \in \mathscr{B}_{x}^{-} \text {and } t>0, \quad \text { then } E \cap(x-t, x) \in \mathscr{B}_{x}^{-} \text {, } \\
& \text { if } E \in \mathscr{B}_{x}^{-} \text {and } F \supset E \text {, then } F \in \mathscr{B}_{x}^{-} .
\end{aligned}
$$


In the end, let

$$
\mathscr{B}_{x}=\mathscr{B}_{x}^{+} \cup \mathscr{B}_{x}^{-} \quad \text { and } \quad \mathscr{B}=\left\{\mathscr{B}_{x}: x \in \mathbb{R}\right\} .
$$

We define the generalized limit numbers of a real function defined in an open interval $I$ similarly as in 3 .

DEFINITION 2. A number $g$ is called a generalized $\mathscr{B}$-limit number of a function $f: I \longrightarrow \mathbb{R}$ at a point $x_{0}$ from the right side if

$$
\{x \in I:|f(x)-g|<\varepsilon\} \in \mathscr{B}_{x_{0}}^{+}
$$

for each positive real number $\varepsilon$.

Infinity (minus infinity) is called a generalized $\mathscr{B}$-limit number of a function $f: I \longrightarrow \mathbb{R}$ at a point $x_{0}$ from the right side if

$$
\{x \in I: f(x)>\varepsilon\} \in \mathscr{B}_{x_{0}}^{+} \quad\left(\{x \in I: f(x)<-\varepsilon\} \in \mathscr{B}_{x_{0}}^{+}\right)
$$

for each positive real number $\varepsilon$.

The set of all generalized $\mathscr{B}$-limit numbers from the right side of a function $f: I \longrightarrow \mathbb{R}$ is denoted by $L_{\mathscr{B}}^{+}(f, x)$.

A number $g$ is called a generalized $\mathscr{B}$-limit number of a function $f: I \longrightarrow \mathbb{R}$ at a point $x_{0}$ from the left side if

$$
\{x \in I:|f(x)-g|<\varepsilon\} \in \mathscr{B}_{x_{0}}^{-}
$$

for each positive real number $\varepsilon$.

Infinity (minus infinity) is called a generalized $\mathscr{B}$-limit number of a function $f: I \longrightarrow \mathbb{R}$ at a point $x_{0}$ from the right side if

$$
\{x \in I: f(x)>\varepsilon\} \in \mathscr{B}_{x_{0}}^{-} \quad\left(\{x \in I: f(x)<-\varepsilon\} \in \mathscr{B}_{x_{0}}^{-}\right)
$$

for each positive real number $\varepsilon$.

The set of all generalized $\mathscr{B}$-limit numbers from the left side of function $f: I \longrightarrow \mathbb{R}$ is denoted by $L_{\mathscr{B}}^{-}(f, x)$.

A number $g$ is called a generalized $\mathscr{B}$-limit number of a function $f: I \longrightarrow \mathbb{R}$ at a point $x_{0}$ if

for each positive real number $\varepsilon$.

$$
\{x \in I:|f(x)-g|<\varepsilon\} \in \mathscr{B}_{x_{0}}
$$

Infinity (minus infinity) is called a generalized $\mathscr{B}$-limit number of a function $f: I \longrightarrow \mathbb{R}$ at a point $x_{0}$ if

$$
\{x \in I: f(x)>\varepsilon\} \in \mathscr{B}_{x_{0}} \quad\left(\{x \in I: f(x)<-\varepsilon\} \in \mathscr{B}_{x_{0}}\right)
$$

for each positive real number $\varepsilon$.

The set of all generalized $\mathscr{B}$-limit numbers of a function $f: I \longrightarrow \mathbb{R}$ is denoted by $L_{\mathscr{B}}(f, x)$. 
It is easy to see that condition (2) implies condition (44). Therefore, every family $\mathfrak{B}$ fulfils all conditions of the family $\mathscr{B}$. Thus, every $\mathfrak{B}$-limit number of any function $f: I \longrightarrow \mathbb{R}$ is $\mathscr{B}$-limit number of that function as well.

There are some examples of classes of sets fulfilling conditions (3) and (4) from Definition 1 but not fulfilling all conditions for classes $\mathfrak{B}$ from the article [3] (see Examples 3 and 5).

One can easily prove Theorem 1 .

TheOREM 1. For every real function $f: I \longrightarrow \mathbb{R}$ and every $x$ from $I$, the sets

$$
L_{\mathscr{B}}^{+}(f, x), \quad L_{\mathscr{B}}^{-}(f, x) \quad \text { and } \quad L_{\mathscr{B}}(f, x)
$$

are closed and

$$
L_{\mathscr{B}}(f, x)=L_{\mathscr{B}}^{+}(f, x) \cup L_{\mathscr{B}}^{-}(f, x) .
$$

P r o of. Equality (77) follows immediately from the definitions of the sets

$$
L_{\mathscr{B}}^{+}(f, x), \quad L_{\mathscr{B}}^{-}(f, x) \quad \text { and } \quad L_{\mathscr{B}}(f, x) .
$$

To prove that the set $L_{\mathscr{B}}^{+}(f, x)$ is closed (in natural topology in the two points compactification of the set of real numbers), it is sufficient to prove that if $g$ is a limit of a sequence $\left(g_{n}\right)_{n=1}^{\infty}$ such that $g_{n} \in L_{\mathscr{B}}^{+}(f, x)$, then $g \in L_{\mathscr{B}}^{+}(f, x)$.

Suppose first that $g \neq \infty$ and $g \neq-\infty$. For every positive $\varepsilon$, there exists a $g_{n}$ such that

$$
\{t \in I:|f(t)-g|<\varepsilon\} \supset\left\{t \in I:\left|f(t)-g_{n}\right|<\frac{\varepsilon}{3}\right\}
$$

and the last set belongs to $L_{\mathscr{B}}^{+}(f, x)$, then the set $\{t \in I:|f(t)-g|<\varepsilon\}$ belongs to $L_{\mathscr{B}}^{+}(f, x)$ as well. It proves that

$$
g \in L_{\mathscr{B}}^{+}(f, x) .
$$

Similar arguments can be used to prove the case when $g$ equals plus or minus infinity.

For example, one of them will be given. Suppose that $g=\infty$. If one of the terms of the sequence $\left(g_{n}\right)_{n=1}^{\infty}$ is equal to $\infty$, then there is nothing to prove. If not, then let $\varepsilon$ be any positive number. Then, there exists a $g_{n}$ such that $g_{n}>\varepsilon+1$ and

$$
\{t \in I: f(t)>\varepsilon\} \supset\left\{t \in I:\left|f(t)-g_{n}\right|<\frac{1}{2}\right\}
$$

and the last set belongs to $L_{\mathscr{B}}^{+}(f, x)$. Hence, the set $\{t \in I: f(t)>\varepsilon\}$ belongs to $L_{\mathscr{B}}^{+}(f, x)$ as well. It proves that

$$
\infty \in L_{\mathscr{B}}^{+}(f, x) .
$$

Quite similar proofs can be done for the set of all generalized $\mathscr{B}$-limit numbers from the left side of the function $f$ and for the set of generalized $\mathscr{B}$-limit numbers of the function $f$. 


\section{GENERALIZED CLUSTER SETS}

Sometimes we consider two kinds of $\mathscr{B}$-limit numbers, then it is convenient to compare two classes fulfilling conditions from Definition 1 and Definition 2.

It is quite obvious in view of definition of $\mathscr{B}$-limit numbers that the following theorem holds, which one can see in its short proof.

THEOREM 2. If two classes $\mathscr{B}_{x}^{+}$and $\mathscr{D}_{x}^{+}$fulfil conditions from Definition 1 , then $\mathscr{B}_{x}^{+} \subset \mathscr{D}_{x}^{+}$if and only if for every function $f: I \longrightarrow \mathbb{R}$ and for each $x \in I$ inclusion $L_{\mathscr{B}}^{+}(f, x) \subset L_{\mathscr{D}}^{+}(f, x)$ holds.

P r o of. The implication

$$
\mathscr{B}_{x}^{+} \subset \mathscr{D}_{x}^{+} \Longrightarrow\left(\forall f: I \rightarrow \mathbb{R} \forall_{x \in I} L_{\mathscr{B}}^{+}(f, x) \subset L_{\mathscr{D}}^{+}(f, x)\right)
$$

is quite obvious.

To prove the converse implication, let us assume that

$$
\mathscr{B}_{x}^{+} \not \subset \mathscr{D}_{x}^{+} \text {. }
$$

Then, there exists a set $E \in \mathscr{B}_{x}^{+} \backslash \mathscr{D}_{x}^{+}$. Consider the characteristic function $f$ of the set $E$. It is evident that

$$
1 \in L_{\mathscr{B}}^{+}(f, x) \wedge 1 \notin L_{\mathscr{D}}^{+}(f, x) .
$$

In this way, we have proved that

$$
\mathscr{B}_{x}^{+} \not \subset \mathscr{D}_{x}^{+} \Longrightarrow\left(\exists_{f: I \rightarrow \mathbb{R}} \exists_{x \in I} L_{\mathscr{B}}^{+}(f, x) \not \subset L_{\mathscr{D}}^{+}(f, x)\right) .
$$

In the same way, one can prove the next theorem

THEOREM 3. If two classes $\mathscr{B}_{x}^{-}$and $\mathscr{D}_{x}^{-}$fulfil conditions from Definition 1 , then $\mathscr{B}_{x}^{-} \subset \mathscr{D}_{x}^{-}$if and only if for every function $f: \mathbb{R} \longrightarrow \mathbb{R}$ and for each $x \in \mathbb{R}$ inclusion $L_{\mathscr{B}}^{-}(f, x) \subset L_{\mathscr{D}}^{-}(f, x)$ holds.

One can see that Theorems 1, 2 and 3 are generalizations of Theorems 2 and 3 from article 3 .

ExAmple 1. Let $\mathscr{B}_{x}^{+}$be the class of sets $E \subset \mathbb{R}$ such that external upper right-sided density of $E$ at $x$ is greater than $\frac{1}{2}$.

It is obvious that this class fulfils conditions (3) and (4). There exist two disjoint measurable sets $A_{1}$ and $A_{2}$ such that

$$
(0, \infty)=A_{1} \cup A_{2}
$$

and density of each of those sets at 0 equals $\frac{1}{2}$. Then, the characteristic function of the set $A_{1}$ has empty set of all right sided $\mathscr{B}$-limit numbers at 0 .

Let us observe that if we add a special condition, we will obtain the next theorem. 


\section{JACEK JĘDRZEJEWSKI — STANISŁAW KOWALCZYK}

THEOREM 4. Let the class $\mathscr{B}_{x}^{+}$fulfil the condition

$$
\text { if } A \cup B \in \mathscr{B}_{x}^{+} \text {, then } A \in \mathscr{B}_{x}^{+} \text {or } B \in \mathscr{B}_{x}^{+} \text {. }
$$

Then, for every function $f: \mathbb{R} \longrightarrow \mathbb{R}$, the set $L_{\mathscr{B}}^{+}(f, x)$ is nonempty.

Proof. Since $f$ is a real function, then at least one of the sets

$$
\{x \in \mathbb{R}: f(x) \leq 0\}, \quad\{x \in \mathbb{R}: f(x) \geq 0\}
$$

belongs to $\mathscr{B}_{x}^{+}$. Assume that the last of those sets belongs to $\mathscr{B}_{x}^{+}$. Let $I_{n}=[n, n+1]$ for every positive integer $n$. Then

$$
[0, \infty)=\bigcup_{n=0}^{\infty} I_{n}
$$

Consider two cases:

(i) there exists a positive integer $n_{0}$ such that $\left\{t \in \mathbb{R}: f(t) \in I_{n_{0}}\right\} \in \mathscr{B}_{x}^{+}$,

(ii) $\left\{t \in \mathbb{R}: f(t) \in I_{n}\right\} \notin \mathscr{B}_{x}^{+}$for each positive integer $n$.

If the second case is fulfilled, then $\infty$ is a right sided $\mathscr{B}$-limit number of the function $f$ at the point $x_{0}$.

If the first case is fulfilled, then at least one of the sets

$$
\left\{t \in \mathbb{R}: f(t) \in\left[n_{0}, n_{0}+\frac{1}{2}\right]\right\}, \quad\left\{t \in \mathbb{R}: f(t) \in\left[n_{0}+\frac{1}{2}, n_{0}+1\right]\right\}
$$

belongs to $\mathscr{B}_{x}^{+}$.

In this manner, we can define a descending sequence of closed intervals $\left(J_{n}\right)_{n=1}^{\infty}$ such that

$$
\left|J_{k}\right|=\frac{1}{2^{k}}, \quad\left\{t \in \mathbb{R}: f(t) \in J_{k}\right\} \in \mathscr{B}_{x}^{+} .
$$

The sequence $\left(J_{k}\right)_{k=1}^{\infty}$ has one point in common, let it be $g$. Then, for every positive number $\varepsilon$, there exists an index $k$ such that

$$
\left\{t: f(t) \in J_{k}\right\} \subset\{t \in \mathbb{R}:|f(t)-g|<\varepsilon\} .
$$

In view of (9), the set $\{t \in \mathbb{R}:|f(t)-g|<\varepsilon\}$ belongs to $\mathscr{B}_{x}^{+}$.

Then $g$ is a right-sided $\mathscr{B}$-limit number of the function $f$ at the point $x$.

Similarly, one can prove

THEOREM 5. Let the class $\mathscr{B}_{x}^{-}$fulfils an additional condition

$$
\text { if } A \cup B \in \mathscr{B}_{x}^{-} \text {, then } A \in \mathscr{B}_{x}^{-} \text {or } B \in \mathscr{B}_{x}^{-} \text {. }
$$

Then, for every function $f: \mathbb{R} \longrightarrow \mathbb{R}$, the sets $L_{\mathscr{B}}^{-}(f, x)$ and $L_{\mathscr{B}}(f, x)$ are nonempty.

Let us remark that the above theorems can be partly completed by 
THEOREM 6. If for every function $f: I \longrightarrow \mathbb{R}$ the sets $L_{\mathscr{B}}^{+}(f, x)$ are nonempty, then the class $\mathscr{B}_{x}^{+}$fulfils an additional condition

$$
A \in \mathscr{B}_{x}^{+} \quad \text { or } \quad I \backslash A \in \mathscr{B}_{x}^{+} \quad \text { for every subset } A \text { of } I .
$$

P r o of. Let us suppose that there exists a subset $A$ of $I$ such that $A \notin \mathscr{B}_{x}^{+}$ and $I \backslash A \notin \mathscr{B}_{x}^{+}$. Then, the characteristic function of the set $A$ has no $\mathscr{B}$-limit number at any point $x$. Thus, the above mentioned condition is fulfilled.

Similarly, one can prove

THEOREM 7. If for every function $f: I \longrightarrow \mathbb{R}$ the sets $L_{\mathscr{B}}^{-}(f, x)$ are nonempty, then the class $\mathscr{B}_{x}^{-}$fulfils an additional condition

$$
A \in \mathscr{B}_{x}^{-} \quad \text { or } \quad I \backslash A \in \mathscr{B}_{x}^{-} \quad \text { for every subset } A \text { of } I .
$$

ExAmple 2. Let $\mathscr{B}_{x}^{+}$be a class of the sets $E$ such that external upper right-sided density of $E$ at the point $x$ is not less than $\frac{1}{2}$.

It is obvious that this class fulfils conditions (3), (4) and (11). There exist three disjoint measurable sets $A_{1}, A_{2}$ and $A_{3}$ such that

$$
(0, \infty)=A_{1} \cup A_{2} \cup A_{3}
$$

and the density at 0 of each of those sets equals $\frac{1}{3}$. Then the function $\chi_{A_{1}}+2 \chi_{A_{2}}$, where $\chi_{A}$ is the characteristic function of the $A$, has an empty set of all right-sided $\mathscr{B}$-limit numbers at 0 .

The previous example proved that condition (11) is not sufficient and the next example will prove that condition (8) is not necessary for nonemptiness of the set of right-sided $\mathscr{B}$-limit numbers of a function.

ExAmple 3 . Let $\mathscr{D}_{x}^{+}$be a class of all sets $E$ such that

$$
\bar{d}_{e}^{+}(E, x)>0 \quad \text { or } \quad\left(E \supset\left\{x+\frac{1}{n} n>n_{0}\right\} \text { for some } n_{0} \in \mathbb{N}\right) .
$$

Moreover, let $\mathscr{B}_{x}^{+}$be a class of all sets $E$ such that

$$
\bar{d}_{e}^{+}(E, x)>0 \text {. }
$$

It is obvious that both of the classes $\mathscr{B}^{+}$and $\mathscr{D}^{+}$fulfil conditions (3) and (4) and the class $\mathscr{B}^{+}$fulfils (8). Theorem 4 implies that $L_{\mathscr{B}}^{+}(f, x) \neq \varnothing$ for each real function $f$. Hence, by Theorem $2, L_{\mathscr{D}}^{+}(f, x) \neq \varnothing$ for each real function $f$.

On the other hand, let

$$
A=\left\{\frac{1}{n}: n>0\right\}, \quad B=\left\{\frac{1}{2 n}: n>0\right\}, \quad C=\left\{\frac{1}{2 n+1}: n>0\right\} .
$$

Hence

$$
A=B \cup C, \quad A \in \mathscr{D}_{0}^{+}, \quad B \notin \mathscr{D}_{0}^{+}, \quad C \notin \mathscr{D}_{0}^{+} .
$$

Thus, condition (8) is not fulfilled. 
Theorems 6 and 7 can be slightly generalized as follows

THEOREM 8. If for every function $f: I \longrightarrow \mathbb{R}$ the sets $L_{\mathscr{B}}^{+}(f, x)$ are nonempty, then for every finite class of subsets of $I$, if $I$ is a union of this class, then at least one of the sets from this class belongs to $\mathscr{B}_{x}^{+}$.

P roof. Let us suppose that there exist subsets $A_{1}, \ldots, A_{n}$ of $\mathbb{R}$ such that $A_{i} \notin \mathscr{B}_{x}^{+}$provided $i \in\{1, \ldots, n\}$. Then, the function $f: \mathbb{R} \longrightarrow \mathbb{R}$, defined by $f(x)=k$ if $x \in A_{k}$ for each $k$ from $\{1, \ldots, n\}$, has no generalized $\mathscr{B}$-limit number at $x$.

Similarly, one can prove

THEOREM 9. If for every function $f: I \longrightarrow \mathbb{R}$ the sets $L_{\mathscr{B}}^{-}(f, x)$ are nonempty, then for every finite class of subsets of $I$ if $I$ is a union of this class, then at least one of the sets from this class belongs to $\mathscr{B}_{x}^{-}$.

\section{Another approach to $\mathscr{B}$-limit numbers}

There is another way of defining generalized $\mathscr{B}$-limit numbers of a function. Let us observe that if there exists a set $E \in \mathscr{B}_{x}^{+}$such that

then $g \in L_{\mathscr{B}}^{+}(f, x)$.

$$
\lim _{t \rightarrow x} f_{l E}(t)=g,
$$

The converse implication is not true, which can be seen in [3]. However

THEOREM 10. Let $f: I \longrightarrow \mathbb{R}$ be arbitrary function and $x \in \mathbb{R}$. Then, conditions

$$
\begin{aligned}
& \qquad g \in L_{\mathscr{B}}^{+}(f, x), \\
& \text { there exists a set } E \in \mathscr{B}_{x}^{+} \text {such that } \lim _{t \rightarrow x} f_{l E}(t)=g,
\end{aligned}
$$

are equivalent if and only if the class $\mathscr{B}$ fulfils the following condition

(W) for every descending sequence of sets $\left(E_{n}\right)_{n=1}^{\infty}$ such that $E_{n} \in \mathscr{B}_{x}^{+}$, there exists a decreasing to $x$ sequence of numbers $\left(x_{n}\right)_{n=1}^{\infty}$ such that

$$
\bigcup_{n=1}^{\infty}\left(E_{n} \cap\left[x_{n+1}, x_{n}\right)\right) \in \mathscr{B}_{x}^{+} .
$$

P r o of. Necessity. Let us suppose that condition (W) is not fulfilled. Then, there exists a sequence of sets $\left(E_{n}\right)_{n=1}^{\infty}$ such that $E_{n} \in \mathscr{B}_{x}^{+}$for every positive integer $n$ and for any decreasing to $x$ sequence $\left(x_{n}\right)_{n=1}^{\infty}$, and

$$
\bigcup_{n=1}^{\infty}\left(E_{n} \cap\left[x_{n+1}, x_{n}\right)\right) \notin \mathscr{B}_{x}^{+} \text {. }
$$


Let us define a function $f$ as follows

$$
f(t)= \begin{cases}\frac{1}{n} & \text { if } t \in E_{n} \backslash E_{n+1} \\ 2 & \text { if } t \notin E_{1}\end{cases}
$$

Then,

$$
f(t) \leq \frac{1}{n} \text { for all } t \in \bigcup_{k=n}^{\infty} E_{k}
$$

Hence, 0 is a generalized $\mathscr{B}$-limit number of the function $f$ at the point $x$.

On the contrary, there exists no set $E$ fulfilling condition (14) from this theorem. In fact, if such a set $E$ existed, then a sequence $\left(t_{n}\right)_{n=1}^{\infty}$ of positive numbers such that

would have existed.

$$
f(t) \leq \frac{1}{n} \quad \text { if } \quad t \in E \cap\left(x, x+t_{n}\right)
$$

The sequence $\left(t_{n}\right)_{n=1}^{\infty}$ could have been chosen to be a decreasing one. Definition of the function $f$ implies

$$
E \cap\left[t_{n+1}, t_{n}\right) \subset E_{n} \cap\left[t_{n+1}, t_{n}\right) \text { and } E \cap\left(x, x+t_{1}\right) \subset \bigcup_{n=1}^{\infty}\left(E_{n} \cap\left[t_{n+1}, t_{n}\right)\right) \text {. }
$$

The last set in the above inclusion does not belong to $\mathscr{B}_{x}^{+}$, which contradicts the choice of the set $E$.

Sufficiency. By the foregoing considerations, it is sufficient to prove that condition (13) implies condition (14).

Let $f: I \longrightarrow \mathbb{R}$ be any function and $g \in L_{\mathscr{B}}^{+}(f, x)$. Let

$$
E_{n}=\left\{x \in I:|f(t)-g|<\frac{1}{n}\right\} \quad \text { if } g \neq \infty \quad \text { and } \quad g \neq-\infty
$$

or

$$
E_{n}=\{x \in I: f(t)>n\} \quad \text { if } g=\infty
$$

or

$$
E_{n}=\{x \in I: f(t)<-n\} \quad \text { if } g=-\infty \text {. }
$$

Then, $E_{n} \in \mathscr{B}_{x}^{+}$for each positive integer $n$. From condition (W), it follows that there exists a sequence $\left(x_{n}\right)_{n=1}^{\infty}$ such that

$$
x<x_{n+1}<x_{n}, \quad \lim _{n \rightarrow \infty} x_{n}=x \quad \text { and } \bigcup_{n=1}^{\infty} E_{n} \cap\left[x_{n+1}, x_{n}\right) \in \mathscr{B}_{x}^{+} .
$$

It is easy to see that if

then

$$
E=\bigcup_{n=1}^{\infty} E_{n} \cap\left[x_{n+1}, x_{n}\right),
$$

$$
\lim _{t \rightarrow x} f_{l E}(t)=g .
$$

This completes the proof. 


\section{Asymmetry}

Definition 3. We say that the class $\mathscr{B}$ fulfils condition $(M)$ if for arbitrary point $x \in \mathbb{R}$, sequence of sets $\left(B_{n}\right)_{n=1}^{\infty}$ and sequence of numbers $\left(x_{n}\right)_{n=1}^{\infty}$ such that

$$
\lim x_{n}=x, \quad x_{n}>x, \quad B_{n} \in \mathscr{B}_{x_{n}}^{+}
$$

the set $\bigcup_{n=1}^{\infty} E_{n}$ belongs to $\mathscr{B}_{x}^{+}$and, moreover, if for arbitrary point $x \in \mathbb{R}$, sequence of sets $\left(B_{n}\right)_{n=1}^{\infty}$ and sequence of numbers $\left(x_{n}\right)_{n=1}^{\infty}$ such that

$$
\lim x_{n}=x, \quad x_{n}<x, \quad B_{n} \in \mathscr{B}_{x_{n}}^{-}
$$

the set $\bigcup_{n=1}^{\infty} E_{n}$ belongs to $\mathscr{B}_{x}^{-}$.

We say that $x$ is a point of asymmetry of the function $f$ if it belongs to the domain of $f$ and $L_{\mathscr{B}}^{+}(f, x) \neq L_{\mathscr{B}}^{-}(f, x)$. If $\mathfrak{B}$ is the class of sets defined in [3, Example 1], then the set of all points of asymmetry of any function is at most countable. It is not true if we consider the class $\mathfrak{B}$ defined in [3], Example 2], see [1] and [7].

THEOREM 11. If the class $\mathscr{B}$ fulfils condition $(M)$, then the set of $\mathscr{B}$-asymmetry points of arbitrary function is at most countable.

P r oof. Let $f: I \longrightarrow \mathbb{R}$ be an arbitrary bounded function. It suffices to prove that the set $A:=\left\{x \in I: L_{\mathscr{B}}^{-}(f, x) \backslash L_{\mathscr{B}}^{+}(f, x) \neq \varnothing\right\}$ is at most countable. Let then $x_{0} \in A$. There exists a number $p$ such that $p \in L_{\mathscr{B}}^{-}\left(f, x_{0}\right) \backslash L_{\mathscr{B}}^{+}\left(f, x_{0}\right)$.

By Theorem 1, there is a positive number $\varepsilon_{0}$ such that

$$
\begin{gathered}
\left(p-\varepsilon_{0}, p+\varepsilon_{0}\right) \cap L_{\mathscr{B}}^{+}\left(f, x_{0}\right)=\varnothing, \\
\left\{x \in I:|f(x)-p|<\varepsilon_{0}\right\} \notin \mathscr{B}_{x_{0}}^{+} \quad \text { and } \quad\left\{x \in I:|f(x)-p|<\varepsilon_{0}\right\} \in \mathscr{B}_{x_{0}}^{-} .
\end{gathered}
$$

Thus, from condition $(M)$, there exist rational numbers $a_{x_{0}}, b_{x_{0}}$ and $c_{x_{0}}$ such that $a_{x_{0}}<p<b_{x_{0}}, \quad c_{x_{0}}>x_{0}, \quad\left\{x \in I: a_{x_{0}}<f(x)<b_{x_{0}}\right\} \notin \mathscr{B}_{x}^{-}$for $x \in\left(x_{0}, c_{x_{0}}\right)$. Let $T: A \longrightarrow \mathbb{Q}^{3}$ assign to each point $x$ from the set $A$ a triple of rational numbers in the above explained manner. It follows that function $T$ is injective. Hence, the set $A$ is at most countable.

If a function $f$ is not bounded then, the function

$$
\phi(x)=\frac{f(x)}{1+|f(x)|}
$$

is bounded and both of them have the same points of asymmetry.

Condition $(M)$ is not necessary to prove that the set of $\mathscr{B}$-asymmetry points of a function is at most countable. For example, see articles [9] and [10]. 


\section{GENERALIZED CLUSTER SETS}

Another problem concerning the asymmetry deals with the set of those points $x$ at which $f(x)$ does not belong to the set of generalized $\mathscr{B}$-limit numbers. Not every class $\mathscr{B}$ is good enough for generalized $\mathscr{B}$-limit numbers to be nonempty. Hence, if one wants to consider the set $\left\{x \in I: f(x) \notin L_{\mathscr{B}}(f, x)\right\}$, it is necessary to be sure that $L_{\mathscr{B}}(f, x)$ is a nonempty set. To get it, it is good to assume the condition from Theorem 4. In this case, it is sufficient to look at article [3, where such a problem has been discussed.

\section{Generalized topology}

Definition 4. For each real $x$, let $\left(\mathscr{B}_{x}^{+}\right)^{*}$ denote the class of nonempty subsets of $\mathbb{R}$ which fulfils the following condition

$$
E \in\left(\mathscr{B}_{x}^{+}\right)^{*} \quad \text { if } \quad \mathbb{R} \backslash E \notin \mathscr{B}_{x}^{+} .
$$

In a very similar way, we define the class $\left(\mathscr{B}_{x}^{-}\right)^{*}$ for each real $x$.

DEFinition 5. For each real $x$, let $\left(\mathscr{B}_{x}^{-}\right)^{*}$ denote the class of nonempty subsets of $\mathbb{R}$ which fulfils the following condition

$$
E \in\left(\mathscr{B}_{x}^{-}\right)^{*} \quad \text { if } \quad \mathbb{R} \backslash E \notin \mathscr{B}_{x}^{-} .
$$

Finally, let us assume that $\left(\mathscr{B}_{x}\right)^{*}=\left(\mathscr{B}_{x}^{+}\right)^{*} \cap\left(\mathscr{B}_{x}^{-}\right)^{*}$ and $\mathscr{B}^{*}=\left\{\left(\mathscr{B}_{x}\right)^{*}: x \in \mathbb{R}\right\}$.

Let us remark that for each topology $\tau$ in the real line, which is stronger than natural topology, the family $\mathfrak{B}_{x}(\tau)$ such that

$$
\mathfrak{B}_{x}^{+}(\tau)=\left\{E \subset \mathbb{R}: \exists_{t>0}\left(x \in \operatorname{cl}_{\tau}(E \cap(x, x+t))\right)\right\}
$$

fulfils conditions (11) and (2) from [3. Thus, each topology stronger than the natural one, defines $\mathfrak{B}$-limit numbers of any real function defined in an interval. There are some classes of sets $\mathfrak{B}$ which cannot be defined by any topology, see in [3, Example 6]. Problems of $\mathscr{B}$-limit numbers of a function are connected in a similar way with generalized topologies. The idea of generalized topological spaces was given by Á. Császár in [2].

Definition 6 ([2]). A family $\Gamma$ of subsets of a nonempty set $X$ is called a generalized topology in $X$ if the following conditions:

(1) $\varnothing \in \Gamma$,

(2) if $U_{s} \in \Gamma$ for $s$ in $S$, then $\bigcup_{s \in S} U_{s} \in \Gamma$, hold.

One can see that generalized topology in a set $X$ does not fulfil the condition (which is required in the case of topology in $X$ ) that the intersection of two sets from generalized topology must belong to that generalized topology. 


\section{JACEK JĘDRZEJEWSKI — STANISŁAW KOWALCZYK}

Definition 7. Let $\Gamma$ be a generalized topology in $\mathbb{R}$. A number $g$ is called $\Gamma$-limit number of a function $f: I \longrightarrow \mathbb{R}$ at a point $x_{0} \in I$ from the right side if

$$
\{x \in I:|f(x)-g|<\varepsilon\} \cap U \cap\left(x_{0}, \infty\right) \neq \varnothing
$$

for each positive real number $\varepsilon$ and $U \in \Gamma$ containing $x_{0}$.

Infinity (minus infinity) is called a generalized $\Gamma$-limit number of a function $f: I \longrightarrow \mathbb{R}$ at a point $x_{0}$ from the right side if

$\{x \in I: f(x)>\varepsilon\} \cap U \cap\left(x_{0}, \infty\right) \neq \varnothing \quad\left(\{x \in I: f(x)<-\varepsilon\} \cap U \cap\left(x_{0}, \infty\right) \neq \varnothing\right)$

for each positive real number $\varepsilon$ and $U \in \Gamma$ containing $x_{0}$.

The set of all generalized $\Gamma$-limit numbers from the right side of a function $f: I \longrightarrow \mathbb{R}$ is denoted by $L_{\Gamma}^{+}(f, x)$.

Similarly, we define when $g$ or $-\infty$ or $\infty$ is $\Gamma$-limit number of a function $f: I \longrightarrow \mathbb{R}$ at a point $x_{0} \in I$ from the left side.

The set of all generalized $\Gamma$-limit numbers from the left side of a function $f: I \longrightarrow \mathbb{R}$ is denoted by $L_{\Gamma}^{-}(f, x)$.

Moreover

Definition 8. Let $\Gamma$ be a generalized topology in $\mathbb{R}$. A number $g$ is called a $\Gamma$-limit number of a function $f: I \longrightarrow \mathbb{R}$ at a point $x_{0}$ if

$$
\{x \in I:|f(x)-g|<\varepsilon\} \cap\left(U \backslash\left\{x_{0}\right\}\right) \neq \varnothing
$$

for each positive real number $\varepsilon$ and $U \in \Gamma$ containing $x_{0}$.

Infinity (minus infinity) is called a generalized $\Gamma$-limit number of a function $f: I \longrightarrow \mathbb{R}$ at a point $x_{0}$ if

$\{x \in I: f(x)>\varepsilon\} \cap\left(U \backslash\left\{x_{0}\right\}\right) \neq \varnothing \quad\left(\{x \in I: f(x)<-\varepsilon\} \cap\left(U \backslash\left\{x_{0}\right\}\right) \neq \varnothing\right)$

for each positive real number $\varepsilon$ and $U \in \Gamma$ containing $x_{0}$.

The set of all generalized $\Gamma$-limit numbers of a function $f: I \longrightarrow \mathbb{R}$ is denoted by $L_{\Gamma}(f, x)$.

THEOREM 12. For each family $\mathscr{B}$, the families

$$
\begin{aligned}
\Gamma_{\mathscr{B}} & :=\left\{U \subset \mathbb{R}: \forall_{x \in U}\left(U \in\left(\mathscr{B}_{x}\right)^{*}\right)\right\}, \\
\Gamma_{\mathscr{B}^{+}} & :=\left\{U \subset \mathbb{R}: \forall_{x \in U}\left(U \in\left(\mathscr{B}_{x}^{+}\right)^{*}\right)\right\}, \\
\Gamma_{\mathscr{B}^{-}} & :=\left\{U \subset \mathbb{R}: \forall_{x \in U}\left(U \in\left(\mathscr{B}_{x}^{-}\right)^{*}\right)\right\}
\end{aligned}
$$

are generalized topologies in $\mathbb{R}$. Moreover,

$$
\Gamma_{\mathscr{B}} \subset \Gamma_{\mathscr{B}^{+}} \cap \Gamma_{\mathscr{B}^{-}} .
$$




\section{GENERALIZED CLUSTER SETS}

Proof. It is obvious that $\varnothing \in \Gamma_{\mathscr{B}}$. Let $\left\{U_{s}: s \in S\right\}$ be a family of sets from $\Gamma_{\mathscr{B}}$ and let $U=\bigcup\left\{U_{s}: s \in S\right\}$. If $x \in U$, then there is $s_{0} \in S$ such that $x \in U_{s_{0}}$. Since $U_{s_{0}} \in \Gamma_{\mathscr{B}}$, we have $U_{s_{0}} \in\left(\mathscr{B}_{x}\right)^{*}$. Thus, $U \in\left(\mathscr{B}_{x}\right)^{*}$ and $U \in \Gamma_{\mathscr{B}}$. In this way we have proved that $\Gamma_{\mathscr{B}}$ is a generalized topology in $\mathbb{R}$.

In the same way, one can prove that $\Gamma_{\mathscr{B}^{+}}$and $\Gamma_{\mathscr{B}^{-}}$are generalized topologies in $\mathbb{R}$.

Take any $U \in \Gamma_{\mathscr{B}}$ and $x \in U$. Then, $U \in\left(\mathscr{B}_{x}\right)^{*}$ by the definition of $\Gamma_{\mathscr{B}}$. Since $\left(\mathscr{B}_{x}\right)^{*}=\left(\mathscr{B}_{x}^{-}\right)^{*} \cap\left(\mathscr{B}_{x}^{+}\right)^{*}$, we have immediately $U \in\left(\mathscr{B}_{x}^{-}\right)^{*}$ and $U \in\left(\mathscr{B}_{x}^{+}\right)^{*}$. Thus $\Gamma_{\mathfrak{B}}=\Gamma_{\mathfrak{B}^{-}} \cap \Gamma_{\mathfrak{B}^{+}}$.

THEOREM 13. For each $f: I \longrightarrow \mathbb{R}$ and each $x \in I$, the inclusions

$$
\begin{aligned}
& L_{\mathscr{B}}^{+}(f, x) \subset L_{\Gamma_{\mathscr{B}}+}(f, x), \\
& L_{\mathscr{B}}^{-}(f, x) \subset L_{\Gamma_{\mathscr{B}}-}(f, x), \\
& L_{\mathscr{B}}(f, x) \subset L_{\Gamma_{\mathscr{B}}}(f, x)
\end{aligned}
$$

hold.

P r o of. Let $g \in L_{\mathscr{B}}^{+}(f, x)$ and assume that $g \notin L_{\Gamma_{\mathscr{B}}^{+}}(f, x)$. Then, there exist $U \in \Gamma_{\mathscr{B}^{+}}$and $\varepsilon>0$ such that

$$
\{t \in I:|f(t)-g|<\varepsilon\} \cap U \cap(x, \infty)=\varnothing .
$$

Since $U \in \Gamma_{\mathscr{B}^{+}}$and $x \in U$, we have $U \in\left(\mathscr{B}_{x}^{+}\right)^{*}$. Thus, $I \backslash U \notin \mathscr{B}_{x}^{+}$.

On the other hand, (20) implies that

Thus

$$
\{t \in I:|f(t)-g|<\varepsilon\} \cap(x, \infty) \subset I \backslash U .
$$

$$
\{t \in I:|f(t)-g|<\varepsilon\} \cap(x, \infty) \notin \mathscr{B}_{x}^{+}
$$

and $g \notin L_{\mathscr{B}}^{+}(f, x)$. The contradiction proves that $g \in L_{\Gamma_{\mathscr{B}}^{+}}(f, x)$.

Now, let $\infty \in L_{\mathscr{B}}^{+}(f, x)$ and assume that $\infty \notin L_{\Gamma_{\mathscr{B}^{+}}}(f, x)$. Then, there exist $U \in \Gamma_{\mathscr{B}^{+}}$and $\varepsilon>0$ such that

$$
\{t \in I: f(t)>\varepsilon\} \cap U \cap(x, \infty)=\varnothing .
$$

Since $U \in \Gamma_{\mathscr{B}^{+}}$and $x \in U$, we have $U \in\left(\mathscr{B}_{x}^{+}\right)^{*}$. Thus $I \backslash U \notin \mathscr{B}_{x}^{+}$.

On the other hand, (5) implies that

Thus

$$
\{t \in I: f(t)>\varepsilon\} \cap(x, \infty) \subset I \backslash U .
$$

$$
\{t \in I: f(t)>\varepsilon\} \cap(x, \infty) \notin \mathscr{B}_{x}^{+}
$$

and $\infty \notin L_{\mathscr{B}}^{+}(f, x)$. The contradiction proves that $\infty \in L_{\Gamma_{\mathscr{B}}^{+}}(f, x)$. 
The proof of implication

is quite similar.

$$
-\infty \in L_{\mathscr{B}}^{+}(f, x) \Longrightarrow-\infty \in L_{\Gamma_{\mathscr{B}}}(f, x)
$$

This remark ends the proof of (17).

The proofs of the remained inclusions are similar.

EXAMPLE 4. Let

and

$$
\mathscr{B}_{x}^{+}=\left\{E \subset \mathbb{R}: \exists_{n_{0}} \forall_{n>n_{0}}\left(x+\frac{1}{n} \in E\right)\right\}
$$

$$
\mathscr{B}_{x}^{-}=\left\{E \subset \mathbb{R}: \exists_{n_{0}} \forall_{n>n_{0}}\left(x-\frac{1}{n} \in E\right)\right\} \quad \text { for each } x \in \mathbb{R} \text {. }
$$

Then,

$$
\left(\mathscr{B}_{x}^{+}\right)^{*}=\left\{E \subset \mathbb{R}: \forall_{n} \exists_{k>n}\left(x+\frac{1}{k} \notin E\right)\right\}
$$

and

$$
\left(\mathscr{B}_{x}^{-}\right)^{*}=\left\{E \subset \mathbb{R}: \forall_{n} \exists_{k>n}\left(x-\frac{1}{k} \notin E\right)\right\} .
$$

Let $A=\left\{\frac{1}{n}: n \in \mathbb{N}_{+}\right\} \cup\left\{-\frac{1}{n}: n \in \mathbb{N}_{+}\right\}$and $f$ be a characteristic function of the set $A$.

It is obvious that $L_{\mathscr{B}}^{+}(f, 0)=L_{\mathscr{B}}^{-}(f, 0)=\{1\}$.

Next, we will show that $0 \in L_{\Gamma_{\mathscr{B}}}(f, 0) \cap L_{\Gamma_{\mathscr{B}^{-}}}(f, 0)$.

Let $\varepsilon$ be a positive number less than 1 and $U$ be a neighbourhood of 0 in $\Gamma_{\mathscr{B}^{+}}$. It is evident that

$$
\{x \in \mathbb{R}:|f(x)-0|<\varepsilon\}=\mathbb{R} \backslash A .
$$

On the other hand, $U \in\left(\mathscr{B}_{0}^{+}\right)^{*}$, and there exists a positive integer $n>1$ such that $\frac{1}{n} \in U$. Now, $U \in\left(\mathscr{B}_{1 / n}^{+}\right)^{*}$. There exists a positive integer $k$ such that $\frac{1}{n}+\frac{1}{k} \in U$ and $\frac{1}{n}+\frac{1}{k}<\frac{1}{n-1}$. Thus,

$$
\frac{1}{n}+\frac{1}{k} \notin A \text { and } \quad\{x \in \mathbb{R}:|f(x)-0|<\varepsilon\} \cap U \neq \varnothing .
$$

In this way we have proved that $0 \in L_{\Gamma_{\mathscr{B}}}(f, 0)$.

In a similar way, one can prove that $0 \in L_{\Gamma_{\mathscr{B}}}(f, 0)$.

It shows that

$$
L_{\mathscr{B}}^{+}(f, 0) \varsubsetneqq L_{\Gamma_{\mathscr{B}}+}(f, 0), \quad L_{\mathscr{B}}^{-}(f, 0) \varsubsetneqq L_{\Gamma_{\mathscr{B}}}(f, 0) .
$$

Definition 9. We say that a family $\mathscr{B}$ fulfils condition $(K)$ if

$$
\forall_{x \in I} \forall_{E \in\left(\mathscr{B}_{x}\right)^{*}, x \in E} \exists_{F \subset E, x \in F} \forall_{t \in F}\left(F \in\left(\mathscr{B}_{t}\right)^{*}\right) \text {. }
$$

Considering generalized topology $\Gamma$, we make use of similar terminology as for topologies. Hence, by generalized interior of a set $A$, we mean the union of all generalized open sets contained in $A$. It is, of course, the largest generalized open set contained in $A$. This set is denoted by $\operatorname{int}_{\Gamma}(A)$. 
THEOREM 14. A family $\mathscr{B}$ fulfils condition $(K)$ if and only if

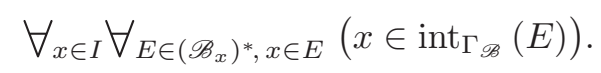

Proof. The proof can be deduced by $x \in \operatorname{int}_{\Gamma_{\mathscr{B}}}(E) \Longleftrightarrow \exists_{F \in \Gamma_{\mathscr{B}}}(x \in F \wedge F \subset E) \Longleftrightarrow \exists_{F \subset E, x \in F} \forall_{t \in F}\left(F \in\left(\mathscr{B}_{t}\right)^{*}\right)$.

THEOREM 15. If a family $\mathscr{B}$ fulfils condition $(K)$, then for each $f: I \longrightarrow \mathbb{R}$ and each $x \in I$ we have

$$
L_{\Gamma_{\mathscr{B}}}(f, x)=L_{\mathscr{B}}(f, x) .
$$

P r o of. The inclusion

$$
L_{\mathscr{B}}(f, x) \subset L_{\Gamma_{\mathscr{B}}}(f, x)
$$

follows from Theorem 13.

To prove the inverse inclusion, we have to consider three cases.

Assume that $g \notin L_{\mathscr{B}}(f, x)$. Then there exists a positive $\varepsilon$ such that

Then

$$
\{t \in I:|f(t)-g|<\varepsilon\} \notin \mathscr{B}_{x} .
$$

$$
\{t \in I:|f(t)-g| \geq \varepsilon\} \in\left(\mathscr{B}_{x}\right)^{*} .
$$

Let $A=\operatorname{int}_{\Gamma_{\mathscr{B}}}(\{t \in I:|f(t)-g| \geq \varepsilon\})$. Thus, $x \in A$, by the condition $(K)$. It implies that

$$
\begin{gathered}
\{t \in I:|f(t)-g|<\varepsilon\} \cap(A \backslash\{x\})=\varnothing, \\
A \in \Gamma_{\mathscr{B}} \quad \text { and } \quad x \in A .
\end{gathered}
$$

So, $g \in L_{\Gamma_{\mathscr{B}}}(f, x)$. In this way, we have proved that

$$
g \in L_{\Gamma_{\mathscr{B}}}(f x) \Longrightarrow g \in L_{\mathscr{B}}(f, x) .
$$

Assume that $\infty \notin L_{\mathscr{B}}(f, x)$. Then, there exists a positive $\varepsilon$ such that

$$
\{t \in I: f(t)>\varepsilon\} \notin \mathscr{B}_{x} .
$$

Then,

$$
\{t \in I: f(t) \leq \varepsilon\} \in\left(\mathscr{B}_{x}\right)^{*} .
$$

Let $A=\operatorname{int}_{\Gamma_{\mathscr{B}}}(\{t \in I:|f(t)-g| \geq \varepsilon\})$. Thus, $x \in A$, by the condition $(K)$. It implies that

$$
\begin{gathered}
\{t \in I: f(t) \geq \varepsilon\} \cap(A \backslash\{x\})=\varnothing, \\
A \in \Gamma_{\mathscr{B}} \quad \text { and } \quad x \in A .
\end{gathered}
$$

In this way, we have proved that

$$
\infty \in L_{\Gamma_{\mathscr{B}}}(f, x) \Longrightarrow \infty \in L_{\mathscr{B}}(f, x) .
$$

The same proof can be repeated for $-\infty$.

In this way, we have proved the following inclusion

which completes the proof.

$$
L_{\Gamma_{\mathscr{B}}}(f, x) \subset L_{\mathscr{B}}(f, x),
$$




\section{JACEK JĘDRZEJEWSKI — STANISŁAW KOWALCZYK}

Next two theorems can be proved in the same way.

THEOREM 16. If a family $\mathscr{B}^{+}$fulfils the following condition

$$
\forall_{x \in I} \forall_{E \in\left(\mathscr{B}_{x}^{+}\right)^{*}} \exists_{F \subset E, x \in F} \forall_{t \in F}\left(F \in\left(\mathscr{B}_{t}^{+}\right)^{*}\right),
$$

then for each $f: I \longrightarrow \mathbb{R}$ and each $x \in I$ we have

$$
L_{\Gamma_{\mathscr{B}}+}(f, x)=L_{\mathscr{B}}^{+}(f, x) .
$$

THEOREM 17. If a family $\mathscr{B}^{-}$fulfils the following condition

$$
\forall_{x \in I} \forall_{E \in\left(\mathscr{B}_{x}^{-}\right)^{*}} \exists_{F \subset E, x \in F} \forall_{t \in F}\left(F \in\left(\mathscr{B}_{t}^{-}\right)^{*}\right),
$$

then for each $f: I \longrightarrow \mathbb{R}$ and each $x \in I$ we have

$$
L_{\Gamma_{\mathscr{B}}-}(f, x)=L_{\mathscr{B}}^{-}(f, x) .
$$

\section{REFERENCES}

[1] BELOWSKA, L.: Résolution d'un probléme de M. Z. Zahorski sur les limites approximatives, Fund. Math. 48 (1960), 277-286.

[2] CSÁSZÁR, Á.: Generalized topology, generalized continuity, Acta Math. Hungar. 96 (2002), 351-357.

[3] JĘDRZEJEWSKI, J. M.: On limit numbers of real functions, Fund. Math. 83 (1973/74), 269-281.

[4] JĘDRZEJEWSKI, J. M.: The generalized limit and generalized continuity, Zeszyty Nauk. Uniw. Łódz. 52 (1973), 19-38.

[5] JĘDRZEJEWSKI, J. M.-WILCZYŃSKI, W.: On the family of sets of limit numbers, Bull. Acad. Pol. Sci. 18 (1970), 453-460.

[6] JĘDRZEJEWSKI, J. M.-WILCZYŃSKI, W.: On the family of sets of $\mathfrak{B}$-limit numbers, Zeszyty Nauk. Uniw. Łódz. 52 (1973), 39-43.

[7] KULBACKA, M.: Sur l'ensemble des points de l'asymmétrie approximative, Acta Sci. Math. (Szeged) 21 (1960), 90-95.

[8] SIERPIŃSKI, W.: Wstęp do Teorii Funkcyj Rzeczywistych, PNW, Warszawa, 1928.

[9] ŚWIAZTKOWSKI, T.: On some generalization of the notion of asymmetry of functions, Colloq. Math. 17 (1967), 17-91.

[10] ŚWIĄTKOWSKI, T.: On a certain generalization of the notion of derivative, Zesz. Nauk. Politech Łódz., Mat. 149 (1972), 89-103.

[11] THOMSON, B. S.: Real Functions, in: Lecture Notes in Math., Vol. 1170, Springer-Verlag, Berlin, 1985.

[12] YOUNG, W. H.: La symétrie de structure des fonctions des variables réelles, Bull. Sci. Math. (2) 52 (1928), 265-280.

[13] WILCZYŃSKI, W.: On the family of sets of approximate limit numbers, Fund. Math. 75 (1972), 169-174. 


\section{GENERALIZED CLUSTER SETS}

[14] WILCZYŃSKI, W.: On the family of sets of qualitative limit numbers, Rev. Roum. Math. Pures Appl. XVIII (1973), 1297-1302.

Received December 2, 2013

\author{
Jacek Jędrzejewski \\ Institute of Mathematics and \\ Computer Science \\ Jan Diugosz University in Częstochowa \\ Al. Armii Krajowej 13/15 \\ PL-42-200 Częstochowa \\ $P O L A N D$ \\ E-mail: jacek.m.jedrzejewski@gmail.com \\ Stanisław Kowalczyk \\ Institute of Mathematics \\ Pomeranian Academy \\ ul. Arciszewskiego $22 b$ \\ PL-76-200 Stupsk \\ POLAND \\ E-mail: stkowalcz@onet.eu
}

